\title{
REGULARIZATION OF AN INDEFINITE ABSTRACT INTERPOLATION PROBLEM WITH A QUADRATIC CONSTRAINT
}

\author{
SANTIAGO GONZALEZ ZERBO, ALEJANDRA MAESTRIPIERI, \\ AND FRANCISCO MARTÍNEZ PERÍA
}

\begin{abstract}
In this work we study an indefinite abstract smoothing problem. After establishing necessary and sufficient conditions for the existence of solutions, the set of admissible parameters is discussed in detail. Then, the relationship of this problem with a linearly constrained interpolation problem is analyzed.
\end{abstract}

\section{INTRODUCTION}

In the sixties, a Hilbert space formulation of spline functions, known as abstract splines, was introduced by M. Atteia [3] and extended by several authors: see e.g. [2, 9, 24, 29]. Abstract splines are the solution to an abstract interpolation problem, which has a natural generalization to Krein spaces. Spline functions in indefinite metric spaces have already been studied in [12, 25] to solve numerical aspects related to learning theory problems. Although the problems presented there are different from those studied in this work, they are closely related.

Given a Hilbert space $\mathcal{H}$ and Krein spaces $\mathcal{K}$ and $\mathcal{E}$, consider (bounded) surjective operators $T: \mathcal{H} \rightarrow \mathcal{K}$ and $V: \mathcal{H} \rightarrow \mathcal{E}$. The abstract interpolation problem in Krein spaces can be stated as follows:

Problem 1.1. Given $z_{0} \in \mathcal{E}$,

$$
\text { minimize }[T x, T x] \text {, subject to }\left[V x-z_{0}, V x-z_{0}\right]=0
$$

and, if the minimum exists, find the set of arguments at which it is attained.

Since $[\cdot, \cdot]_{\mathcal{K}}$ and $[\cdot, \cdot]_{\mathcal{E}}$ are indefinite inner products, the above one is a quadratically constrained quadratic programming (QCQP) problem, where the objective function $x \mapsto[T x, T x]$ is not convex, while the function defining the equality constraint $x \mapsto\left[V x-z_{0}, V x-z_{0}\right]$ is sign indefinite.

2020 Mathematics Subject Classification. Primary 46C20; Secondary 47B50, 65D10.

Key words and phrases. abstract splines; Krein spaces; quadratically constrained quadratic programming. 
Given $x_{0} \in \mathcal{H}$ such that $V x_{0}=z_{0}$, the condition $\left[V x-z_{0}, V x-z_{0}\right]=0$ becomes $x \in x_{0}+\mathcal{C}_{V}$, where $\mathcal{C}_{V}=\{u \in \mathcal{H}:[V u, V u]=0\}$ is the set of neutral elements of the quadratic form $x \mapsto[V x, V x]$. Hence, 11.1) can be restated as: Analyze the existence of

$$
\min _{x \in x_{0}+\mathcal{C}_{V}}[T x, T x]
$$

and, if the minimum exists, find the set of arguments at which it is attained.

If $V^{\#} V$ is a positive (or negative) semidefinite operator in $\mathcal{H}$ then $\mathcal{C}_{V}$ coincides with $N(V)$, and $(1.1)$ becomes the interpolation problem previously studied in [17. But, if $V^{\#} V$ is indefinite, the set $\mathcal{C}_{V}$ is strictly larger than $N(V)$. Therefore, in the main part of this work $V^{\#} V$ is assumed to be indefinite, i.e. neither positive nor negative semidefinite. The particular case of a semidefinite $V^{\#} V$ is only considered in Section 4

One of the main drawbacks for tackling this QCQP problem is that $\mathcal{C}_{V}$ is not a convex set. Moreover, the convex hull of $\mathcal{C}_{V}$ is the complete Hilbert space $\mathcal{H}$, thus replacing $\mathcal{C}_{V}$ by its convex hull trivializes the problem.

The classical penalization approach known as the Tikhonov regularization can be applied to this problem in order to obtain an associated optimization problem over the Hilbert space $\mathcal{H}$. Indeed, the aim of this work is to study the following generalization of the abstract smoothing problem (see [4]):

Problem. Given $\rho \in \mathbb{R} \backslash\{0\}$ and a fixed $z_{0} \in \mathcal{E}$, analyze the existence of

$$
\min _{x \in \mathcal{H}}\left([T x, T x]_{\mathcal{K}}+\rho\left[V x-z_{0}, V x-z_{0}\right]_{\mathcal{E}}\right)
$$

and, if the minimum exists, find the set of arguments at which it is attained.

The advantage of the above regularized problem is that it can be restated as an indefinite least-squares problem. These problems have been thoroughly studied before, both in finite-dimensional spaces [20, 21, 30, 13, 22, 27, 8] and in infinite dimensional Krein spaces [7, 18, 19. Although the existence of solutions to the above Problem as well as descriptions of the set of solutions can be derived from the corresponding indefinite least-squares problem, it is desirable to reformulate these conditions in terms of the original data $T, V, \rho$ and $z_{0}$.

The indefinite abstract smoothing problem in Krein spaces was initially studied in [17], but with a linear constraint. Also, in [11] another version of this abstract smoothing problem was studied, but instead of minimizing $F_{\rho}(x)=[T x, T x]_{\mathcal{K}}+$ $\rho\left\|V x-z_{0}\right\|_{\mathcal{E}}^{2}$, the authors were interested in stabilizing (i.e. finding the critical points of) this function.

The contents of the paper are organized as follows. Section 2 presents the notation, and it also contains a compilation of the basic terminology and results on Krein spaces used along this work. Section 3 deals with the indefinite abstract smoothing problem. After establishing necessary and sufficient conditions for the existence of solutions to this problem, the set of admissible parameters is discussed in detail. Finally, Section 4 is devoted to analyzing the close relationship between the indefinite abstract smoothing problem and a particular version of the indefinite 
abstract interpolation problem, where the quadratic constraint in 1.1 is replaced by a linear constraint. This problem coincides with the one previously considered in [17. We focus our attention on those situations in which a set of interpolating splines is also a subset of the solutions to a certain smoothing interpolation problem, or, even further, in which an indefinite interpolation problem can be posed as an indefinite smoothing problem, and viceversa.

\section{Preliminaries}

Along this work $\mathcal{H}$ denotes a complex (separable) Hilbert space. If $\mathcal{K}$ is another Hilbert space then $\mathcal{L}(\mathcal{H}, \mathcal{K})$ is the vector space of bounded linear operators from $\mathcal{H}$ into $\mathcal{K}$ and $\mathcal{L}(\mathcal{H})=\mathcal{L}(\mathcal{H}, \mathcal{H})$ stands for the algebra of bounded linear operators in $\mathcal{H}$.

If $T \in \mathcal{L}(\mathcal{H}, \mathcal{K})$ then $R(T)$ stands for the range of $T$ and $N(T)$ for its nullspace. The Moore-Penrose inverse $T^{\dagger}$ of an operator $T \in \mathcal{L}(\mathcal{H}, \mathcal{K})$ is the densely defined (not necessarily bounded) linear operator

$$
T^{\dagger}: R(T) \dot{+} R(T)^{\perp} \rightarrow \mathcal{H}
$$

defined as follows: for $y \in R(T)$ and $z \in R(T)^{\perp}, T^{\dagger}(y+z)=x$, where $x \in N(T)^{\perp}$ is the (unique) vector that satisfies $T x=y$.

Recall that the Moore-Penrose inverse $T^{\dagger}$ is bounded if and only if $T$ has a closed range. For detailed expositions on the Moore-Penrose inverse, see [6, 26].

The following well-known result about the product of closed range operators [10, 23] is frequently used along the paper.

Proposition 2.1. Given Hilbert spaces $\mathcal{H}_{1}, \mathcal{H}_{2}$ and $\mathcal{K}$, let $A \in \mathcal{L}\left(\mathcal{K}, \mathcal{H}_{2}\right)$ and $B \in \mathcal{L}\left(\mathcal{H}_{1}, \mathcal{K}\right)$ have closed ranges. Then, $A B \in \mathcal{L}\left(\mathcal{H}_{1}, \mathcal{H}_{2}\right)$ has closed range if and only if $R(B)+N(A)$ is closed in $\mathcal{K}$.

2.1. Krein spaces. In what follows we present the standard notation and some basic results on Krein spaces. For a complete exposition on the subject (and the proofs of the results below) see [7, [5, 1, 15, 28.

An indefinite inner product space $(\mathcal{F},[\cdot, \cdot])$ is a (complex) vector space $\mathcal{F}$ endowed with a Hermitian sesquilinear form $[\cdot, \cdot]: \mathcal{F} \times \mathcal{F} \rightarrow \mathbb{C}$.

A vector $x \in \mathcal{F}$ is positive, negative or neutral if $[x, x]>0,[x, x]<0$ or $[x, x]=0$, respectively. The set of positive vectors in $\mathcal{F}$ is denoted by $\mathcal{P}^{++}(\mathcal{F})$, and the set of nonnegative vectors in $\mathcal{F}$ by $\mathcal{P}^{+}(\mathcal{F})$. The sets of negative, nonpositive and neutral vectors in $\mathcal{F}$ are defined analogously, and they are denoted by $\mathcal{P}^{--}(\mathcal{F})$, $\mathcal{P}^{-}(\mathcal{F})$ and $\mathcal{P}^{0}(\mathcal{F})$, respectively.

Likewise, a subspace $\mathcal{M}$ of $\mathcal{F}$ is positive if every $x \in \mathcal{M}, x \neq 0$, is a positive vector in $\mathcal{F}$; and it is nonnegative if $[x, x] \geq 0$ for every $x \in \mathcal{M}$. Negative, nonpositive and neutral subspaces are defined mutatis mutandis.

If $\mathcal{S}$ is a subset of an indefinite inner product space $\mathcal{F}$, the orthogonal companion to $\mathcal{S}$ is defined by

$$
\mathcal{S}^{[\perp]}=\{x \in \mathcal{F}:[x, s]=0 \text { for every } s \in \mathcal{S}\} .
$$

It is easy to see that $\mathcal{S}^{[\perp]}$ is always a subspace of $\mathcal{F}$. 
Definition 2.2. An indefinite inner product space $(\mathcal{H},[\cdot, \cdot])$ is a Krein space if it can be decomposed as a direct (orthogonal) sum of a Hilbert space and an anti Hilbert space, i.e. there exist subspaces $\mathcal{H}_{ \pm}$of $\mathcal{H}$ such that $\left(\mathcal{H}_{+},[\cdot, \cdot]\right)$ and $\left(\mathcal{H}_{-},-[\cdot, \cdot]\right)$ are Hilbert spaces,

$$
\mathcal{H}=\mathcal{H}_{+} \dot{+} \mathcal{H}_{-},
$$

and $\mathcal{H}_{+}$is orthogonal to $\mathcal{H}_{-}$with respect to the indefinite inner product. Sometimes we use the notation $[\cdot, \cdot]_{\mathcal{H}}$ instead of $[\cdot, \cdot]$ to emphasize the Krein space considered.

A pair of subspaces $\mathcal{H}_{ \pm}$as in 2.1) is called a fundamental decomposition of $\mathcal{H}$. Given a Krein space $\mathcal{H}$ and a fundamental decomposition $\mathcal{H}=\mathcal{H}_{+} \dot{+} \mathcal{H}_{-}$, the direct (orthogonal) sum of the Hilbert spaces $\left(\mathcal{H}_{+},[\cdot, \cdot]\right)$ and $\left(\mathcal{H}_{-},-[\cdot, \cdot]\right)$ is denoted by $(\mathcal{H},\langle\cdot, \cdot\rangle)$.

If $\mathcal{H}=\mathcal{H}_{+} \dot{+} \mathcal{H}_{-}$and $\mathcal{H}=\mathcal{H}_{+}^{\prime} \dot{+} \mathcal{H}_{-}^{\prime}$ are two different fundamental decompositions of $\mathcal{H}$, the corresponding associated inner products $\langle\cdot, \cdot\rangle$ and $\langle\cdot, \cdot\rangle^{\prime}$ turn out to be equivalent on $\mathcal{H}$. Therefore, the norm topology on $\mathcal{H}$ does not depend on the chosen fundamental decomposition.

If $\left(\mathcal{H},[\cdot, \cdot]_{\mathcal{H}}\right)$ and $\left(\mathcal{K},[\cdot, \cdot]_{\mathcal{K}}\right)$ are Krein spaces, $\mathcal{L}(\mathcal{H}, \mathcal{K})$ stands for the vector space of linear transformations which are bounded with respect to any of the associated Hilbert spaces $\left(\mathcal{H},\langle\cdot, \cdot\rangle_{\mathcal{H}}\right)$ and $\left(\mathcal{K},\langle\cdot, \cdot\rangle_{\mathcal{K}}\right)$. Given $T \in \mathcal{L}(\mathcal{H}, \mathcal{K})$, the adjoint operator of $T$ (in the Krein spaces sense) is the unique operator $T^{\#} \in \mathcal{L}(\mathcal{K}, \mathcal{H})$ such that

$$
[T x, y]_{\mathcal{K}}=\left[x, T^{\#} y\right]_{\mathcal{H}}, \quad x \in \mathcal{H}, y \in \mathcal{K} .
$$

We will frequently use that if $T \in \mathcal{L}(\mathcal{H}, \mathcal{K})$ and $\mathcal{M}$ is a closed subspace of $\mathcal{K}$ then

$$
T^{\#}(\mathcal{M})^{[\perp]_{\mathcal{H}}}=T^{-1}\left(\mathcal{M}^{[\perp]_{\mathcal{K}}}\right) .
$$

Given a subspace $\mathcal{M}$ of a Krein space $\mathcal{H}$, the isotropic part of $\mathcal{M}$ is defined by $\mathcal{M}^{\circ}:=\mathcal{M} \cap \mathcal{M}^{[\perp]}$. Then, $\mathcal{M}$ is nondegenerate if $\mathcal{M}^{\circ}=\{0\}$.

A subspace $\mathcal{M}$ of a Krein space $\mathcal{H}$ is pseudo-regular if $\mathcal{M}+\mathcal{M}^{[\perp]}$ is a closed subspace of $\mathcal{H}$, and it is regular if $\mathcal{M}+\mathcal{M}^{[\perp]}=\mathcal{H}$. Regular subspaces are examples of nondegenerate subspaces, but pseudo-regular subspaces can be degenerate ones. However, if $\mathcal{M}$ is a pseudo-regular subspace then there exists a regular subspace $\mathcal{R}$ such that

$$
\mathcal{M}=\mathcal{M}^{\circ}[\dot{+}] \mathcal{R}
$$

where $[\dot{+}]$ stands for the direct orthogonal sum with respect to the indefinite inner product $[\cdot, \cdot]$. In fact, any closed subspace $\mathcal{R}$ such that $\mathcal{M}=\mathcal{M}^{\circ} \dot{+} \mathcal{R}$ satisfies 2.3 and turns out to be a regular subspace of $\mathcal{H}$ (see e.g. [16]). Note that a subspace $\mathcal{M}$ is regular if and only if it is pseudo-regular and nondegenerate.

The following propositions can be found in [19, Lemma 3.4] and [5, Chapter 1, $\S 7$, respectively.

Proposition 2.3. Given Krein spaces $\mathcal{H}$ and $\mathcal{K}$, let $T \in \mathcal{L}(\mathcal{H}, \mathcal{K})$ with closed range. Then, $R(T)$ is pseudo-regular if and only if $R\left(T^{\#} T\right)$ is closed. 
A subspace $\mathcal{M}$ of a Krein space $(\mathcal{H},[\cdot, \cdot])$ is uniformly positive if there exists $\alpha>0$ such that

$$
[x, x] \geq \alpha\|x\|^{2} \quad \text { for every } x \in \mathcal{M}
$$

where $\|\cdot\|$ is the norm of any associated Hilbert space. Uniformly negative subspaces are defined mutatis mutandis.

Proposition 2.4. Let $\mathcal{M}$ be a subspace of a Krein space $\mathcal{H}$. Then, $\mathcal{M}$ is closed and uniformly positive (resp. negative) if and only if $\mathcal{M}$ is regular and nonegative (resp. nonpositive).

The following result, taken from [5, Chapter 1, §1], deals with indefinite inner product spaces which are not necessarily Krein spaces. It will be one of the main tools to study the problem posed in 1.1 .

Lemma 2.5. Suppose that $\left(\mathcal{F}_{1},[\cdot, \cdot]_{1}\right)$ is an indefinite inner product space and $\left(\mathcal{F}_{2},[\cdot, \cdot]_{2}\right)$ is an arbitrary inner product space. If the linear mapping $T: \mathcal{F}_{1} \rightarrow \mathcal{F}_{2}$ satisfies $T\left(\mathcal{P}_{1}^{0}\right) \subset \mathcal{P}_{2}^{+}$then, for every $y \in \mathcal{P}_{1}^{--}$and $z \in \mathcal{P}_{1}^{++}$,

$$
\frac{[T y, T y]_{2}}{[y, y]_{1}} \leq \frac{[T z, T z]_{2}}{[z, z]_{1}}
$$

Moreover, under these conditions,

$$
\mu_{+}(T):=\inf _{z \in \mathcal{P}_{1}^{++}} \frac{[T z, T z]_{2}}{[z, z]_{1}} \quad \text { and } \quad \mu_{-}(T):=\sup _{y \in \mathcal{P}_{1}^{--}} \frac{[T y, T y]_{2}}{[y, y]_{1}}
$$

exist, $\mu_{-}(T) \leq \mu_{+}(T)$ and, for any $\mu \in\left[\mu_{-}(T), \mu_{+}(T)\right]$, the following inequality holds:

$$
[T x, T x]_{2} \geq \mu[x, x]_{1} \quad \text { for every } x \in \mathcal{F}_{1} .
$$

Observe that if $0 \in\left[\mu_{-}, \mu_{+}\right]$then $T^{\#} T$ is a positive semidefinite operator.

\section{INDEFINITE SMOOTHING SPLINES}

From now on, $\left(\mathcal{E},[\cdot, \cdot]_{\mathcal{E}}\right)$ and $\left(\mathcal{K},[\cdot, \cdot]_{\mathcal{K}}\right)$ are Krein spaces and $\mathcal{H}$ is a Hilbert space, $T \in L(\mathcal{H}, \mathcal{K})$ and $V \in L(\mathcal{H}, \mathcal{E})$ are two given surjective operators, and $V^{\#} V$ is assumed to be indefinite.

Consider the Tikhonov regularization of the problem posed in 1.1:

Problem 3.1. Given $\rho \in \mathbb{R}$ and fixed $z_{0} \in \mathcal{E}$, analyze the existence of

$$
\min _{x \in \mathcal{H}}\left([T x, T x]_{\mathcal{K}}+\rho\left[V x-z_{0}, V x-z_{0}\right]_{\mathcal{E}}\right)
$$

and, if the minimum exists, find the set of arguments at which it is attained.

Hereafter, we address it as the indefinite abstract smoothing problem.

As mentioned in the Introduction, Problem 3.1 can be restated as an indefinite least-squares problem. Define an indefinite inner product on $\mathcal{K} \times \mathcal{E}$ by:

$$
\left[(y, z),\left(y^{\prime}, z^{\prime}\right)\right]_{\rho}=\left[y, y^{\prime}\right]_{\mathcal{K}}+\rho\left[z, z^{\prime}\right]_{\mathcal{E}}, \quad y, y^{\prime} \in \mathcal{K} \text { and } z, z^{\prime} \in \mathcal{E} .
$$


It is easy to see that the space $\left(\mathcal{K} \times \mathcal{E},[\cdot, \cdot]_{\rho}\right)$ is a Krein space if and only if $\rho \neq 0$. Also, defining the operator $L: \mathcal{H} \rightarrow \mathcal{K} \times \mathcal{E}$ by

$$
L x=(T x, V x), \quad x \in \mathcal{H},
$$

it is immediate that Problem 3.1 is equivalent to the following:

Problem 3.2. Given $\rho \in \mathbb{R}$ and a fixed $z_{0} \in \mathcal{E}$, analyze the existence of

$$
\min _{x \in \mathcal{H}}\left[L x-\left(0, z_{0}\right), L x-\left(0, z_{0}\right)\right]_{\rho}
$$

and, if the minimum exists, find the set of arguments at which it is attained.

Indefinite least-squares problems have been thoroughly studied before: see e.g. [8, 18, 19] and the references therein. It is well known that, if $\rho \neq 0$, there exists a solution to (3.1) if and only if $R(L)$ is nonnegative in $\left(\mathcal{K} \times \mathcal{E},[\cdot, \cdot]_{\rho}\right)$ and $\left(0, z_{0}\right) \in$ $R(L)+R(L)^{[\perp]}$; see [7, Thm. 8.4]. In this case,

$$
\tilde{x} \in \mathcal{H} \text { is a solution to Problem } 3.2 \quad \Longleftrightarrow \quad L \tilde{x}-\left(0, z_{0}\right) \in R(L)^{[\perp]} .
$$

Moreover, if $\tilde{x}$ is a particular solution, the set of solutions is the affine manifold

$$
\tilde{x}+N\left(L^{\#} L\right),
$$

where $L^{\#}$ stands for the adjoint operator of $L$ (see the Preliminaries).

Next, we show that the existence of a constant $\rho \neq 0$ such that $R(L)$ is a nonnegative subspace of $\left(\mathcal{K} \times \mathcal{E},[\cdot, \cdot]_{\rho}\right)$ is completely determined by the action of $T$ on the vectors of $\mathcal{C}_{V}$.

Theorem 3.3. The following conditions are equivalent:

(1) $T\left(\mathcal{C}_{V}\right) \subseteq \mathcal{P}^{+}(\mathcal{K})$;

(2) there exists $\rho \in \mathbb{R}$ such that $T^{\#} T+\rho V^{\#} V \in \mathcal{L}(\mathcal{H})^{+}$.

Proof. By Lemma 2.5 if $T\left(\mathcal{C}_{V}\right) \subseteq \mathcal{P}^{+}(\mathcal{K})$ then there exists $\mu \in \mathbb{R}$ such that

$$
[T x, T x]_{\mathcal{K}} \geq \mu[V x, V x]_{\mathcal{E}} \quad \text { for every } x \in \mathcal{H} .
$$

But this is equivalent to saying that $T^{\#} T-\mu V^{\#} V \in \mathcal{L}(\mathcal{H})^{+}$. Thus, (2) holds considering $\rho=-\mu$.

Conversely, suppose that $T^{\#} T+\rho V^{\#} V \in \mathcal{L}(\mathcal{H})^{+}$for some $\rho \in \mathbb{R}$. Then, for every $x \in \mathcal{C}_{V}$,

$$
0 \leq\left\langle\left(T^{\#} T+\rho V^{\#} V\right) x, x\right\rangle=[T x, T x]+\rho[V x, V x]=[T x, T x] .
$$

If $T\left(\mathcal{C}_{V}\right) \subseteq \mathcal{P}^{+}(\mathcal{K})$, then Lemma 2.5 states that

$$
\rho_{-}:=-\inf _{\{x:[V x, V x]>0\}} \frac{[T x, T x]}{[V x, V x]} \text { and } \rho_{+}:=-\sup _{\{x:[V x, V x]<0\}} \frac{[T x, T x]}{[V x, V x]}
$$

exist, and $\rho_{-} \leq \rho_{+}$. Furthermore, $T^{\#} T+\rho V^{\#} V \in \mathcal{L}(\mathcal{H})^{+}$if and only if $\rho \in$ $\left[\rho_{-}, \rho_{+}\right]$. Since $\left(\mathcal{K} \times \mathcal{E},[\cdot, \cdot]_{\rho}\right)$ is a Krein space if and only if $\rho \neq 0$, the argument above leads to the following corollary. 
Corollary 3.4. Assume that $T\left(\mathcal{C}_{V}\right) \subseteq \mathcal{P}^{+}(\mathcal{K})$, and consider $\rho_{ \pm}$defined by 3.2 . If $\rho \neq 0$ then the following conditions are equivalent:

(1) $T^{\#} T+\rho V^{\#} V \in \mathcal{L}(\mathcal{H})^{+}$;

(2) $\rho \in\left[\rho_{-}, \rho_{+}\right]$;

(3) $R(L)$ is a nonnegative subspace of $\left(\mathcal{K} \times \mathcal{E},[\cdot, \cdot]_{\rho}\right)$.

Proof. The equivalence between (1) and (2) is derived from Lemma 2.5

To establish their equivalence with (3), let us calculate the adjoint operator of $L$ : given $x \in \mathcal{H}$ and $(y, z) \in \mathcal{K} \times \mathcal{E}$,

$$
\begin{aligned}
{[L x,(y, z)]_{\rho} } & =[T x, y]_{\mathcal{K}}+\rho[V x, z]_{\mathcal{E}}=\left\langle x, T^{\#} y\right\rangle+\rho\left\langle x, V^{\#} z\right\rangle \\
& =\left\langle x, T^{\#} y+\rho V^{\#} z\right\rangle .
\end{aligned}
$$

Hence,

$$
L^{\#}(y, z)=T^{\#} y+\rho V^{\#} z, \quad(y, z) \in \mathcal{K} \times \mathcal{E},
$$

and it is immediate that $L^{\#} L=T^{\#} T+\rho V^{\#} V$. Therefore, $[L x, L x]_{\rho} \geq 0$ for every $x \in \mathcal{H}$ if and only if $T^{\#} T+\rho V^{\#} V \in \mathcal{L}(\mathcal{H})^{+}$.

From now on, we assume that $T\left(\mathcal{C}_{V}\right) \subseteq \mathcal{P}^{+}(\mathcal{K})$. In this case there exists an interval of admissible parameters $\left[\rho_{-}, \rho_{+}\right]$such that $T^{\#} T+\rho V^{\#} V \in \mathcal{L}(\mathcal{H})^{+}$if $\rho \in\left[\rho_{-}, \rho_{+}\right]$. If $\rho_{-}=\rho_{+}=0$ then Problem 3.1 becomes trivial. Therefore, we also assume that $\rho_{-} \neq 0$ or $\rho_{+} \neq 0$, and we consider a fixed $\rho \in\left[\rho_{-}, \rho_{+}\right], \rho \neq 0$.

Rewriting the discussion after Problem 3.2 in terms of the original data $T, V$ and $\rho$, the following characterization of the existence of solutions to the indefinite abstract smoothing problem is obtained; see also [17, Lemma 4.3].

Theorem 3.5. Given $z_{0} \in \mathcal{E}$, Problem 3.1 admits a solution if and only if $T^{\#} T+$ $\rho V^{\#} V \in \mathcal{L}(\mathcal{H})^{+}$and $V^{\#} z_{0} \in R\left(T^{\#} T+\rho V^{\#} V\right)$.

Proof. As mentioned above, Problem 3.1 admits a solution if and only if $R(L)$ is nonnegative and $\left(0, z_{0}\right) \in R(L)+R(L)^{[\perp]}$. Theorem 3.3 shows that $R(L)$ is nonnegative if and only if $L^{\#} L=T^{\#} T+\rho V^{\#} V \in \mathcal{L}(\mathcal{H})^{+}$.

Also, $\left(0, z_{0}\right) \in R(L)+R(L)^{[\perp]}$ if and only if there exists $\tilde{x} \in \mathcal{H}$ such that

$$
\left[L \tilde{x}-\left(0, z_{0}\right), L x\right]_{\rho}=0 \quad \text { for every } x \in \mathcal{H} \text {, }
$$

or equivalently, $L^{\#}(L \tilde{x})=L^{\#}\left(0, z_{0}\right)=\rho V^{\#} z_{0}$. Thus,

$$
\left(0, z_{0}\right) \in R(L)+R(L)^{[\perp]} \quad \text { if and only if } \quad V^{\#} z_{0} \in R\left(T^{\#} T+\rho V^{\#} V\right) .
$$

Definition 3.6. Any element $\tilde{x} \in \mathcal{H}$ that is a solution to Problem 3.1 is called a $(T, V, \rho)$-smoothing spline to $z_{0} \in \mathcal{E}$. The set of $(T, V, \rho)$-smoothing splines to $z_{0}$ is denoted by $\operatorname{sm}\left(T, V, \rho, z_{0}\right)$.

Since $T$ and $V$ are fixed along this work, $\operatorname{sm}\left(T, V, \rho, z_{0}\right)$ is shortened to $\operatorname{sm}\left(\rho, z_{0}\right)$.

Corollary 3.7. Assume that $\operatorname{sm}\left(\rho, z_{0}\right) \neq \varnothing$ for a vector $z_{0} \in \mathcal{E}$. Then, $\tilde{x} \in$ $\operatorname{sm}\left(\rho, z_{0}\right)$ if and only if $\tilde{x}$ is a solution to the equation

$$
\left(T^{\#} T+\rho V^{\#} V\right) x=\rho V^{\#} z_{0} .
$$


In this case,

$$
\operatorname{sm}\left(\rho, z_{0}\right)=\tilde{x}+N\left(T^{\#} T+\rho V^{\#} V\right) .
$$

If the conditions established in Theorem 3.5 are satisfied, then

$$
\tilde{x}:=\rho\left(T^{\#} T+\rho V^{\#} V\right)^{\dagger} V^{\#} z_{0} \in \operatorname{sm}\left(\rho, z_{0}\right) .
$$

If $T^{\#} T+\rho V^{\#} V \in \mathcal{L}(\mathcal{H})^{+}$, the smoothing problem admits a solution for those $z_{0} \in \mathcal{E}$ such that $V^{\#} z_{0} \in R\left(L^{\#} L\right)$, i.e. the set of admissible points for the indefinite abstract smoothing problem is

$$
\mathcal{A}:=\left(V^{\#}\right)^{-1}\left(R\left(L^{\#} L\right)\right) .
$$

It is easy to see that the set of admissible points is closed if and only if the subspace $R\left(L^{\#} L\right) \cap R\left(V^{\#}\right)$ is closed in $\mathcal{H}$.

Since the inclusion $\left(V^{\#}\right)^{-1}\left(R\left(L^{\#} L\right)\right) \subseteq V\left(N\left(L^{\#} L\right)\right)^{[\perp]}$ holds, a necessary condition in order to have a well-posed problem is that

$$
z_{0} \in V\left(N\left(T^{\#} T+\rho V^{\#} V\right)\right)^{[\perp]},
$$

i.e. that the set of admissible points $\mathcal{A}$ is contained in $V\left(N\left(T^{\#} T+\rho V^{\#} V\right)\right)^{[\perp]}$.

The following lemmas lead to characterizing the case in which the set of admissible points coincides with this closed subspace.

Lemma 3.8. Let $(y, z) \in \mathcal{K} \times \mathcal{E}$. Then, $(y, z) \in \overline{R(L)+R(L)^{[\perp]}}$ if and only if

$$
z-V T^{\dagger} y \in V\left(N\left(T^{\#} T+\rho V^{\#} V\right)\right)^{[\perp]} \text {. }
$$

Proof. First, we prove that

$$
R(L)^{\circ}=L\left(N\left(L^{\#} L\right)\right) .
$$

In fact, the inclusion $L\left(N\left(L^{\#} L\right)\right) \subseteq R(L) \cap N\left(L^{\#}\right)=R(L)^{\circ}$ is straightforward. To obtain the other inclusion, it suffices to apply $L$ to both sides of the inclusion $L^{-1}\left(N\left(L^{\#}\right)\right) \subseteq N\left(L^{\#} L\right)$.

Using that $T^{\#}$ is injective and $\left(T^{\#}\right)^{\dagger}=\left(T^{\dagger}\right)^{\#}$, it is easy to see that $\left(T^{\#} T+\right.$ $\left.\rho V^{\#} V\right) x=0$ if and only if $T x=-\rho\left(T^{\dagger}\right)^{\#} V^{\#} V x$. Hence,

$$
R(L)^{\circ}=\left\{\left(-\rho\left(T^{\dagger}\right)^{\#} V^{\#} V x, V x\right) \in \mathcal{K} \times \mathcal{E}: x \in N\left(L^{\#} L\right)\right\} .
$$

Now, since $\overline{R(L)+R(L)^{[\perp]}}=\left(R(L)^{\circ}\right)^{[\perp]}$, the above description of $R(L)^{\circ}$ implies that $(y, z) \in \overline{R(L)+R(L)^{[\perp]}}$ if and only if

$$
\left[(y, z),\left(-\rho\left(T^{\dagger}\right)^{\#} V^{\#} V x, V x\right)\right]_{\rho}=0 \quad \text { for every } x \in N\left(L^{\#} L\right) .
$$

But $\left[(y, z),\left(-\rho\left(T^{\dagger}\right)^{\#} V^{\#} V x, V x\right)\right]_{\rho}=\left[y,-\rho\left(T^{\dagger}\right)^{\#} V^{\#} V x\right]_{\mathcal{K}}+\rho[z, V x]_{\mathcal{E}}=\rho[z-$ $\left.V T^{\dagger} y, V x\right]_{\mathcal{E}}$. Hence, $(y, z) \in \overline{R(L)+R(L)^{[\perp]}}$ if and only $\left[z-V T^{\dagger} y, V x\right]_{\mathcal{E}}=0$ for every $x \in N\left(L^{\#} L\right)$. Therefore, the assertion is proved.

Lemma 3.9. The following conditions are equivalent:

(1) $R(L)$ is a closed subspace of $\left(\mathcal{K} \times \mathcal{E},[\cdot, \cdot]_{\rho}\right)$;

(2) $T(N(V))$ is a closed subspace of $\mathcal{K}$;

(3) $N(T)+N(V)$ is a closed subspace of $\mathcal{H}$. 
Proof. (1) $\rightarrow(2)$ : Assume that $R(L)$ is a closed subspace. Then, since $L(N(V))=$ $T(N(V)) \times\{0\}, T(N(V))$ is closed if and only if $L(N(V))$ is closed, or equivalently, if $N(V)+N(L)$ is closed; see Proposition 2.1. But the last condition is fulfilled because $N(L)=N(T) \cap N(V) \subseteq N(V)$.

The equivalence between (2) and (3) also follows from Proposition 2.1. $(3) \rightarrow(1)$ : Recall that $N(T)+N(V)$ is closed if and only if $N(T)^{\perp}+N(V)^{\perp}$ is closed; see [14, Lemma 11]. If we assume that $N(T)+N(V)$ is closed, then $R\left(L^{\#}\right)=$ $N(T)^{\perp}+N(V)^{\perp}$ is closed, and consequently $R(L)$ is closed.

We are now in a position to characterize the case in which the set of admissible points coincides with the subspace $V\left(N\left(L^{\#} L\right)\right)^{[\perp]}$.

Proposition 3.10. Assume that $T^{\#} T+\rho V^{\#} V \in \mathcal{L}(\mathcal{H})^{+}$. Then, the following conditions are equivalent:

(1) $\mathcal{A}=V\left(N\left(T^{\#} T+\rho V^{\#} V\right)\right)^{[\perp]}$;

(2) $R(L)+R(L)^{[\perp]}$ is a closed subspace of $\left(\mathcal{K} \times \mathcal{E},[\cdot, \cdot]_{\rho}\right)$.

Proof. Assume that $\mathcal{A}=V\left(N\left(L^{\#} L\right)\right)^{[\perp]}$. Given $(y, z) \in \overline{R(L)+R(L)^{[\perp]}}$, Lemma 3.8 implies that $z-V T^{\dagger} y \in \mathcal{A}$, or equivalently, the indefinite least-squares problem consisting of minimizing $[L x-(y, z), L x-(y, z)]$ over $x \in \mathcal{H}$ admits a solution. In this case $(y, z) \in R(L)+R(L)^{[\perp]}$; see [7, Thm. 8.4]. Therefore, $R(L)+R(L)^{[\perp]}$ is closed.

Conversely, assume that $R(L)+R(L)^{[\perp]}$ is closed. First, the inclusion $\mathcal{A} \subseteq$ $V\left(N\left(L^{\#} L\right)\right)^{[\perp]}$ always holds. To see the other inclusion, if $z_{0} \in V\left(N\left(L^{\#} L\right)\right)^{[\perp]}$ then, by Lemma 3.8 it follows that $\left(0, z_{0}\right) \in R(L)+R(L)^{[\perp]}$, i.e. $\operatorname{sm}\left(\rho, z_{0}\right) \neq \varnothing$. Hence, $z_{0} \in \mathcal{A}$.

As an immediate corollary, we get the following characterization of the conditions under which $R(L)$ is a pseudo-regular subspace.

Corollary 3.11. Assume that $T^{\#} T+\rho V^{\#} V \in \mathcal{L}(\mathcal{H})^{+}$and $N(T)+N(V)$ is a closed subspace of $\mathcal{H}$. Then, the following conditions are equivalent:

(1) $\operatorname{sm}\left(\rho, z_{0}\right) \neq \varnothing$ for every $z_{0} \in V\left(N\left(T^{\#} T+\rho V^{\#} V\right)\right)^{[\perp]}$;

(2) $R(L)$ is a pseudo-regular subspace of $\left(\mathcal{K} \times \mathcal{E},[\cdot, \cdot]_{\rho}\right)$;

(3) $T^{\#} T+\rho V^{\#} V$ has closed range.

Proof. First of all, note that Lemma 3.9 implies that $R(L)$ is a closed subspace. The equivalence between (1) and (2) then follows from Proposition 3.10 , and applying Proposition 2.3 yields the equivalence between (2) and (3).

The following proposition describes necessary and sufficient conditions for the existence of smoothing splines to every element of $\mathcal{E}$ (see [17, Prop. 4.5]).

Proposition 3.12. Assume that $T^{\#} T+\rho V^{\#} V \in \mathcal{L}(\mathcal{H})^{+}$. Then, the following conditions are equivalent:

(1) $\operatorname{sm}\left(\rho, z_{0}\right) \neq \varnothing$ for every $z_{0} \in \mathcal{E}$;

(2) $R(L)$ is a regular subspace of $\left(\mathcal{K} \times \mathcal{E},[\cdot, \cdot]_{\rho}\right)$; 
(3) $R\left(T^{\#} T+\rho V^{\#} V\right)=N(T)^{\perp}+N(V)^{\perp}$.

In this case, if $\widetilde{x} \in \operatorname{sm}\left(\rho, z_{0}\right)$ then

$$
\operatorname{sm}\left(\rho, z_{0}\right)=\widetilde{x}+N(T) \cap N(V) .
$$

Proof. (1) $\rightarrow(3)$ : If $\operatorname{sm}\left(\rho, z_{0}\right) \neq \varnothing$ for every $z_{0} \in \mathcal{E}$, then $\left(V^{\#}\right)^{-1}\left(R\left(L^{\#} L\right)\right)=\mathcal{E}$. This implies that $R\left(V^{\#} V\right)=R\left(V^{\#}\right) \subseteq R\left(L^{\#} L\right)$. Therefore, $R\left(L^{\#} L\right)=R\left(T^{\#} T\right)+$ $R\left(V^{\#} V\right)=N(T)^{\perp}+N(V)^{\perp}$.

$(3) \rightarrow(2)$ : If $R\left(L^{\#} L\right)=N(T)^{\perp}+N(V)^{\perp}=R\left(L^{\#}\right)$, taking the counterimage by $L^{\#}$ to both sides of the equality gives

$$
\mathcal{H}=\left(L^{\#}\right)^{-1}\left(R\left(L^{\#} L\right)\right)=R(L)+N\left(L^{\#}\right)=R(L)+R(L)^{[\perp]} .
$$

Therefore, $R(L)$ is regular.

$(2) \rightarrow(1)$ : If $R(L)+R(L)^{[\perp]}=\mathcal{H}$, applying $L^{\#}$ to both sides of the equality we get $R\left(L^{\#} L\right)=R\left(L^{\#}\right)=N(T)^{\perp}+N(V)^{\perp}$. Then,

$$
\left(V^{\#}\right)^{-1}\left(R\left(L^{\#} L\right)\right) \supseteq\left(V^{\#}\right)^{-1}\left(N(V)^{\perp}\right)=\mathcal{E} .
$$

Therefore, $\operatorname{sm}\left(\rho, z_{0}\right) \neq \varnothing$ for every $z_{0} \in \mathcal{E}$.

\section{Relationship With The LinEARly CONSTRAined INDEFINITE ABStRACT INTERPOLATION PROBLEM}

In this section a particular version of the constrained interpolation problem formulated in Problem 1.1 is analyzed - one which is closely related to the smoothing problem that arises from its regularization. If the quadratic constraint in (1.1) is replaced by a linear constraint, then Problem 1.1 translates into the following:

Problem 4.1. Given $z_{0} \in \mathcal{E}$,

$$
\text { minimize }[T x, T x] \text {, subject to } V x=z_{0}
$$

and, if the minimum exists, find the set of arguments at which it is attained.

Note that Problem 1.1 in fact reduces to this problem if $V^{\#} V$ is (positive or negative) semidefinite.

This problem was already considered in [17] where, under some hypotheses, it was proved that the set of solutions to Problem 4.1 for a given $z_{0} \in \mathcal{E}$ coincides with $\operatorname{sm}\left(\rho, z_{0}^{\prime}\right)$ for another $z_{0}^{\prime} \in \mathcal{E}$. Hereafter, we propose a deeper insight in this relationship. In particular, we study situations in which the solutions to Problem 4.1 are contained in a set of $(T, V, \rho)$-smoothing splines, and the cases when Problem 4.1 can be translated into an abstract indefinite smoothing problem and viceversa.

Definition 4.2. Any element $\tilde{x} \in \mathcal{H}$ that is a solution to Problem 4.1 is called a $(T, V)$-interpolating spline to $z_{0} \in \mathcal{E}$. The set of $(T, V)$-interpolating splines to $z_{0}$ is denoted by $\operatorname{sp}\left(T, V, z_{0}\right)$. 
Since $T$ and $V$ are fixed along this work, $\operatorname{sp}\left(T, V, z_{0}\right)$ is shortened to $\operatorname{sp}\left(z_{0}\right)$.

Necessary and sufficient conditions for the existence of solutions to Problem 4.1 are given in the following proposition, which is an immediate consequence of [17, Lemma 3.4].

Proposition 4.3. Assume that $T(N(V))$ is nonnegative and let $z_{0} \in \mathcal{E}$. Then, the following conditions are equivalent:

(1) $\operatorname{sp}\left(z_{0}\right) \neq \varnothing$;

(2) $z_{0} \in V\left(T^{\#} T(N(V))^{\perp}\right)$.

In this case, $s p\left(z_{0}\right)$ is an affine manifold parallel to the subspace

$$
\mathcal{N}_{0}:=N(V) \cap T^{\#} T(N(V))^{\perp} .
$$

Remark 4.4. Note that [17, Lemma 3.4] ensures that, given $z_{0} \in \mathcal{E}, x_{0} \in \operatorname{sp}\left(z_{0}\right)$ if and only if $V x_{0}=z_{0}$ and $x_{0} \in T^{\#} T(N(V))^{\perp}$.

In [17, Prop. 3.8] necessary and sufficient conditions for the existence of interpolating splines to every vector of the space were provided: $\operatorname{sp}\left(z_{0}\right) \neq \varnothing$ for every $z_{0} \in \mathcal{E}$ if and only if $T(N(V))$ is a (closed) uniformly positive subspace of $\mathcal{K}$. This is the case if $R(L)$ is a (positive) regular subspace of $\left(\mathcal{K} \times \mathcal{E},[\cdot, \cdot]_{\rho}\right)$; see [17, Thm. 4.7]. Also, several properties of the subspace $T(N(V))$ were analyzed in order to characterize the interpolating splines.

In the following we proceed to study a more general case. With this aim, we first describe the relationship between the subspaces $R(L)$ and $T(N(V))$.

Lemma 4.5. The following conditions hold:

(1) $T(N(V))^{\circ}=T\left(\mathcal{N}_{0}\right)$. Moreover,

$$
T(N(V))=T\left(\mathcal{N}_{0}\right)[\dot{+}] T\left(N(V) \ominus \mathcal{N}_{0}\right) ;
$$

(2) $T(N(V))$ is nondegenerate if and only if $\mathcal{N}_{0}=N(V) \cap N(T)$;

(3) $T(N(V))$ is pseudo-regular if and only if $N(V)+T^{\#} T(N(V))^{\perp}$ is a closed subspace of $\mathcal{H}$. In this case, $T\left(N(V) \ominus \mathcal{N}_{0}\right)$ is regular;

(4) $T(N(V))$ is regular if and only if $\mathcal{H}=N(V)+T^{\#} T(N(V))^{\perp}$.

Proof. Most of the conditions in the statement can be derived from

$$
T^{\#} T(N(V))^{\perp}=T^{-1}\left(T(N(V))^{[\perp]}\right),
$$

which is a consequence of 2.2 in the Preliminaries.

(1) If $y \in T(N(V))^{\circ}$, there exists $x \in N(V)$ such that $y=T x \in T\left(N(V)^{[\perp]}\right)$. So, 4.2 implies that $x \in \mathcal{N}_{0}$ and $y=T x \in T\left(\mathcal{N}_{0}\right)$. The other inclusion is also a consequence of 4.2 .

Also, since $N(V)=\mathcal{N}_{0} \oplus\left(N(V) \ominus \mathcal{N}_{0}\right)$ it is easy to see that $T(N(V))=T\left(\mathcal{N}_{0}\right)+$ $T\left(N(V) \ominus \mathcal{N}_{0}\right)$. Moreover, these subspaces are $[\cdot, \cdot]_{\mathcal{K}}$-orthogonal because $T\left(\mathcal{N}_{0}\right) \subseteq$ $T(N(V))^{[\perp]}$ and $T\left(N(V) \ominus \mathcal{N}_{0}\right) \subseteq T(N(V))$. It remains to prove that the sum is direct, but if $y \in T\left(\mathcal{N}_{0}\right) \cap T\left(N(V) \ominus \mathcal{N}_{0}\right)$ then there exist $x_{1} \in \mathcal{N}_{0}$ and $x_{2} \in N(V) \ominus$ $\mathcal{N}_{0}$ such that $T x_{1}=y=T x_{2}$. So, $x_{2}-x_{1} \in N(V) \cap N(T) \subseteq \mathcal{N}_{0}$ and $x_{2}=\left(x_{2}-\right.$ $\left.x_{1}\right)+x_{1} \in \mathcal{N}_{0} \cap\left(N(V) \ominus \mathcal{N}_{0}\right)=\{0\}$. Thus, $T(N(V))=T\left(\mathcal{N}_{0}\right)[\dot{+}] T\left(N(V) \ominus \mathcal{N}_{0}\right)$. 
(2) Observe that $N(V) \cap N(T) \subseteq \mathcal{N}_{0}$. Also, $T(N(V))$ is nondegenerate if and only if $T\left(\mathcal{N}_{0}\right)=\{0\}$, or equivalently, if $\mathcal{N}_{0} \subseteq N(T)$. This completes the proof of the assertion.

(3) Assume that $T(N(V))+T(N(V))^{[\perp]}$ is closed in $\mathcal{K}$. Since $T$ is bounded,

$$
\begin{aligned}
T^{-1}\left(T(N(V))+T(N(V))^{[\perp]}\right) & =T^{-1}(T(N(V)))+T^{-1}\left(T(N(V))^{[\perp]}\right) \\
& =(N(V)+N(T))+T^{\#} T(N(V))^{\perp} \\
& =N(V)+T^{\#} T(N(V))^{\perp}
\end{aligned}
$$

is also a closed subspace.

Conversely, assume that $N(V)+T^{\#} T(N(V))^{\perp}$ is closed in $\mathcal{H}$. Let $\left(y_{n}\right)_{n \in \mathbb{N}}$ be a sequence in $T(N(V))+T(N(V))^{[\perp]}$ such that $y_{n} \rightarrow y$ for some $y \in \mathcal{K}$. Since $T$ is surjective, there exists $x \in N(T)^{\perp}$ such that $T x=y$. Also, by (4.3), for each $n \geq 1$ there exists $x_{n} \in N(V)+T^{\#} T(N(V))^{\perp}$ such that $T x_{n}=y_{n}$. Since $T^{\dagger}$ is bounded,

$$
P_{N(T) \perp} x_{n}=T^{\dagger} T x_{n}=T^{\dagger} y_{n} \rightarrow T^{\dagger} y=T^{\dagger} T x=x .
$$

Since $N(T) \subseteq T^{\#} T(N(V))^{\perp}$ it is immediate that $N(T)+\left(N(V)+T^{\#} T(N(V))^{\perp}\right)$ is a closed subspace. Equivalently, $P_{N(T)^{\perp}}\left(N(V)+T^{\#} T(N(V))^{\perp}\right)$ is also closed in $\mathcal{H}$. By 4.4 , it follows that $x \in P_{N(T)^{\perp}}\left(N(V)+T^{\#} T(N(V))^{\perp}\right)$. So, there exists $z \in N(V)+T^{\#} T(N(V))^{\perp}$ such that $x=P_{N(T)^{\perp}} z$. Thus, $y=T x=T P_{N(T) \perp z}=$ $T z \in T(N(V))+T(N(V))^{[\perp]}$ (see (4.3)). Therefore, $T(N(V))+T(N(V))^{[\perp]}$ is closed in $\mathcal{K}$.

It remains to show that if $T(N(V))$ is pseudo-regular then $T\left(N(V) \ominus \mathcal{N}_{0}\right)$ is regular. Since $T\left(\mathcal{N}_{0}\right)=T(N(V))^{\circ}$ and 4.1$)$, it suffices to prove that $T\left(N(V) \ominus \mathcal{N}_{0}\right)$ is closed.

By Proposition 2.1, $T\left(N(V) \ominus \mathcal{N}_{0}\right)$ is closed if and only if $\left(N(V) \ominus \mathcal{N}_{0}\right)+N(T)$ is closed. By 4.3), $N(V)+T^{\#} T(N(V))^{\perp}=\left(N(V) \ominus \mathcal{N}_{0}\right) \oplus T^{\#} T(N(V))^{\perp}$ is closed. Since $N(T) \subseteq T^{\#} T(N(V))^{\perp}$, then $\left(N(V) \ominus \mathcal{N}_{0}\right)+N(T)$ is also closed (and regular). Thus, the proof is complete.

(4) Assume that $T(N(V))$ is regular. Then, 4.3 implies that

$$
\mathcal{H}=T^{-1}(\mathcal{K})=T^{-1}\left(T(N(V))+T(N(V))^{[\perp]}\right)=N(V)+T^{\#} T(N(V))^{\perp} .
$$

Conversely, assume that $\mathcal{H}=N(V)+T^{\#} T(N(V))^{\perp}$. Since $T$ is surjective,

$$
\begin{aligned}
\mathcal{K} & =T\left(N(V)+T^{\#} T(N(V))^{\perp}\right)=T(N(V))+T\left(T^{-1}\left(T(N(V))^{[\perp]}\right)\right) \\
& =T(N(V))+T(N(V))^{[\perp]},
\end{aligned}
$$

i.e. $T(N(V))$ is regular.

To continue describing the relationship between the subspaces $R(L)$ and $T(N(V))$, we present the following lemma and proposition.

Lemma 4.6. If $R(L)$ is a (closed) nonnegative subspace of $\left(\mathcal{K} \times \mathcal{E},[\cdot, \cdot]_{\rho}\right)$ then $T(N(V))$ is a (closed) positive subspace of $\mathcal{K}$. In particular, $T(N(V))$ is nondegenerate. 
Proof. Assume that $R(L)$ is a closed nonnegative subspace of $\left(\mathcal{K} \times \mathcal{E},[\cdot, \cdot]_{\rho}\right)$. By Lemma 3.9. $T(N(V))$ is a closed subspace of $\mathcal{K}$. Also, since $T(N(V)) \times\{0\}=$ $L(N(V))$ is a subspace of $R(L)$, it follows that $T(N(V))$ is nonnegative.

In order to prove that $T(N(V))$ is positive, it is sufficient to show that $T(N(V))$ is nondegenerate. So, let $x \in N(V)$ be such that $T x \in T(N(V))^{\circ}$. Since $x \in N(V)$,

$$
0=[T x, T x]=\langle L x, L x\rangle .
$$

Since $L^{\#} L \in L(\mathcal{H})^{+}$(see Theorem 3.3 it follows that $T^{\#} T x=L^{\#} L x=0$. Finally, the surjectivity of $T$ implies the injectivity of $T^{\#}$ and, in particular, $T x=0$.

Proposition 4.7. If $R(L)$ is a nonnegative pseudo-regular subspace of $\left(\mathcal{K} \times \mathcal{E},[\cdot, \cdot]_{\rho}\right)$ then $T(N(V))$ is a (closed) uniformly positive subspace of $\mathcal{K}$.

Proof. The idea of the proof is to show that $T(N(V)) \times\{0\}$ is contained in a regular complement of $R(L)^{\circ}$ in $R(L)$. First, observe that $R(L)^{\circ}$ is a neutral subspace and that, by Lemma 4.6, $T(N(V)) \times\{0\}$ is a closed positive subspace. Hence, $R(L)^{\circ} \cap(T(N(V)) \times\{0\})=\{0\}$. Also, $T(N(V)) \times\{0\}=L(N(V))$ is $[\cdot, \cdot]_{\rho^{-}}$ orthogonal to $R(L)^{\circ}$. Therefore, there exists a closed subspace $\mathcal{M}$ of $R(L)$ such that

$$
R(L)=R(L)^{\circ}[\dot{+}] \mathcal{M} \text { and } T(N(V)) \times\{0\} \subseteq \mathcal{M} .
$$

Since $R(L)$ is pseudo-regular and nonnegative, the subspace $\mathcal{M}$ is a uniformly positive subspace of $\mathcal{K} \times \mathcal{E}$ and $T(N(V)) \times\{0\}$ has the same property (by transitivity). Thus, $T(N(V))$ is uniformly positive.

Combining [17, Prop. 3.8] with the above result, we can now ensure the existence of $(T, V)$-interpolating splines to every element of $\mathcal{E}$ in case that $R(L)$ is a (nonnegative) pseudo-regular subspace.

Corollary 4.8. If $R(L)$ is a nonnegative pseudo-regular subspace of $\left(\mathcal{K} \times \mathcal{E},[\cdot, \cdot]_{\rho}\right)$, then

$$
\operatorname{sp}\left(z_{0}\right) \neq \varnothing \quad \text { for every } z_{0} \in \mathcal{E}
$$

We now focus our attention on the situations in which a set of interpolating splines is also a subset of the solutions to a certain smoothing interpolation problem, or, even further, in which an interpolating splines problem can be posed as a smoothing problem, and viceversa. We start by presenting what is known so far; see [17, Thm. 4.7].

Proposition 4.9. Suppose that $R(L)$ is a positive regular subspace of $\left(\mathcal{K} \times \mathcal{E},[\cdot, \cdot]_{\rho}\right)$. Then, for every $z_{0} \in \mathcal{E}$ there exists $w_{0} \in \mathcal{E}$ such that

$$
\operatorname{sp}\left(w_{0}\right)=\operatorname{sm}\left(\rho, z_{0}\right) .
$$

Although this result is interesting (it says that every $(T, V)$-interpolating spline is a $(T, V, \rho)$-smoothing spline and viceversa), the hypothesis under consideration is quite strong. The rest of the manuscript is devoted to some intermediate results by relaxing the conditions imposed on $R(L)$. 
Proposition 4.10. Suppose that $R(L)$ is a nonnegative subspace of $\left(\mathcal{K} \times \mathcal{E},[\cdot, \cdot]_{\rho}\right)$. Then, the following conditions hold:

(1) for every $z_{0} \in \mathcal{E}$ such that $\operatorname{sm}\left(\rho, z_{0}\right) \neq \varnothing$ there exists $w_{0} \in \mathcal{E}$ such that

$$
\varnothing \neq s p\left(w_{0}\right) \subseteq \operatorname{sm}\left(\rho, z_{0}\right) ;
$$

(2) for every $w_{0} \in \mathcal{E}$ such that $\operatorname{sp}\left(w_{0}\right) \neq \varnothing$ there exists $z_{0} \in \mathcal{E}$ such that

$$
\operatorname{sp}\left(w_{0}\right) \subseteq \operatorname{sm}\left(\rho, z_{0}\right) .
$$

Proof. (1) Assume that $x_{0} \in \operatorname{sm}\left(\rho, z_{0}\right)$. By Proposition 3.7 it follows that $\left(T^{\#} T+\right.$ $\left.\rho V^{\#} V\right) x_{0}=\rho V^{\#} z_{0}$. Since $T^{\#} T x_{0}=V^{\#}\left(\rho z_{0}-V x_{0}\right) \in N(V)^{\perp}$, we have that $x_{0} \in T^{\#} T(N(V))^{\perp}$.

Also, Lemma 4.6 implies that $T(N(V))$ is a positive and nondegenerate subspace of $\mathcal{K}$. But then $N(V) \cap T^{\#} T(N(V))^{\perp}=N(V) \cap N(T)$; see Lemma 4.5. Setting $w_{0}:=V x_{0}$, by Remark 4.4 it follows that $x_{0} \in s p\left(w_{0}\right)$, and thus

$$
s p\left(w_{0}\right)=x_{0}+N(V) \cap N(T) .
$$

Finally, $s p\left(w_{0}\right)=x_{0}+N(V) \cap N(T) \subseteq x_{0}+N\left(L^{\#} L\right)=s m\left(\rho, z_{0}\right)$.

(2) Note that $T(N(V))$ is a nonnegative subspace of $\mathcal{K}$ because $R(L)$ is a nonnegative subspace of $\left(\mathcal{K} \times \mathcal{E},[,]_{\rho}\right)$. Indeed, $L(N(V))=T(N(V)) \times\{0\}$.

Assume that $s p\left(w_{0}\right) \neq \varnothing$ for some $w_{0} \in \mathcal{E}$. By Remark 4.4, there exists $x_{0} \in$ $T^{\#} T(N(V))^{\perp}$ such that $V x_{0}=w_{0}$. Since $T^{\#} T x_{0} \in N(V)^{\perp}=R\left(V^{\#}\right)$, it follows that $T^{\#} T x_{0}=V^{\#}\left(V^{\#}\right)^{\dagger} T^{\#} T x_{0}$. Then,

$$
\left(T^{\#} T+\rho V^{\#} V\right) x_{0}=\rho V^{\#}\left(\frac{1}{\rho}\left(V^{\#}\right)^{\dagger} T^{\#} T+V\right) x_{0} .
$$

Thus, denoting $z_{0}:=\left(\frac{1}{\rho}\left(V^{\#}\right)^{\dagger} T^{\#} T+V\right) x_{0}$, Corollary 3.7 ensures that $x_{0} \in$ $\operatorname{sm}\left(\rho, z_{0}\right)$. Since $N(V) \cap N(T) \subseteq N\left(L^{\#} L\right)$, the result follows.

Note that given $z_{0} \in \mathcal{E}$ such that $\operatorname{sm}\left(\rho, z_{0}\right) \neq \varnothing$, by 3.3 , setting $w_{0}:=$ $\rho V\left(T^{\#} T+\rho V^{\#} V\right)^{\dagger} V^{\#} z_{0}$ yields $\varnothing \neq s p\left(w_{0}\right) \subseteq s m\left(\rho, z_{0}\right)$. Analogously, given $w_{0} \in \mathcal{E}$ such that $s p\left(w_{0}\right) \neq \varnothing$, if $x_{0} \in T^{\#} T(N(V))^{\perp}$ is such that $w_{0}=V x_{0}$, then setting $z_{0}:=\left(\frac{1}{\rho}\left(V^{\#}\right)^{\dagger} T^{\#} T+V\right) x_{0}$ yields $\operatorname{sp}\left(w_{0}\right) \subseteq \operatorname{sm}\left(\rho, z_{0}\right)$.

As an immediate consequence of Proposition 4.10, we obtain the following corollary.

Corollary 4.11. Suppose that $R(L)$ is a positive nondegenerate subspace of $(\mathcal{K} \times$ $\left.\mathcal{E},[\cdot, \cdot]_{\rho}\right)$. Then, the following conditions hold:

(1) for every $z_{0} \in \mathcal{E}$ such that $\operatorname{sm}\left(\rho, z_{0}\right) \neq \varnothing$ there exists $w_{0} \in \mathcal{E}$ such that

$$
\varnothing \neq s p\left(w_{0}\right)=\operatorname{sm}\left(\rho, z_{0}\right) ;
$$

(2) for every $w_{0} \in \mathcal{E}$ such that $s p\left(w_{0}\right) \neq \varnothing$ there exists $z_{0} \in \mathcal{E}$ such that

$$
\operatorname{sp}\left(w_{0}\right)=\operatorname{sm}\left(\rho, z_{0}\right) .
$$

Proof. By (3.4), $R(L)^{\circ}=L\left(N\left(L^{\#} L\right)\right)$. Hence, $R(L)$ is nondegenerate if and only $N\left(L^{\#} L\right) \subseteq N(L)=N(T) \cap N(V)$. In this case, $N\left(L^{\#} L\right)=N(T) \cap N(V)$, and the statement then follows from Proposition 4.10 


\section{REFERENCES}

[1] T. Ando, Linear operators on Krě̆n spaces, Hokkaido University, Research Institute of Applied Electricity, Division of Applied Mathematics, Sapporo, 1979. MR 0560903

[2] P. M. Anselone and P. J. Laurent, A general method for the construction of interpolating or smoothing spline-functions, Numer. Math. 12 (1968), 66-82. MR 0249904.

[3] M. Atteia, Généralisation de la définition et des propriétés des "spline fonctions", C. R. Acad. Sci. Paris 260 (1965), 3550-3553. MR 0212469

[4] M. Atteia, Hilbertian kernels and spline functions, Studies in Computational Mathematics, 4, North-Holland, Amsterdam, 1992. MR 1205348

[5] T. Ya. Azizov and I. S. Iokhvidov, Linear operators in spaces with an indefinite metric, translated from the Russian by E. R. Dawson, Pure and Applied Mathematics (New York), John Wiley \& Sons, Chichester, 1989. MR 1033489.

[6] A. Ben-Israel and T. N. E. Greville, Generalized inverses, second edition, CMS Books in Mathematics/Ouvrages de Mathématiques de la SMC, 15, Springer-Verlag, New York, 2003. MR 1987382

[7] J. Bognár, Indefinite inner product spaces, Ergebnisse der Mathematik und ihrer Grenzgebiete, Band 78, Springer-Verlag, New York, 1974. MR 0467261

[8] A. Bojanczyk, N. J. Higham and H. Patel, Solving the indefinite least squares problem by hyperbolic QR factorization, SIAM J. Matrix Anal. Appl. 24 (2003), no. 4, 914-931. MR 2003312

[9] C. de Boor, Convergence of abstract splines, J. Approx. Theory 31 (1981), no. 1, 80-89. MR 0619809

[10] R. Bouldin, The product of operators with closed range, Tohoku Math. J. (2) 25 (1973), 359-363. MR 0326424

[11] S. Canu, C.S. Ong, and X. Mary, Splines with non positive kernels, in More progresses in analysis. Proceedings of the 5th international ISAAC congress (Catania, Italy, 2005), 163-173, Hackensack, NJ, World Scientific, 2009.

[12] S. Canu, C.S. Ong, X. Mary, and A. Smola, Learning with non-positive kernels, in Proc. 21st International Conference on Machine Learning (Banff, Canada, 2004), 639-646, New York, Association for Computing Machinery, 2004.

[13] S. Chandrasekaran, M. Gu and A. H. Sayed, A stable and efficient algorithm for the indefinite linear least-squares problem, SIAM J. Matrix Anal. Appl. 20 (1999), no. 2, 354-362. MR 1646860

[14] F. Deutsch, The angle between subspaces of a Hilbert space, in Approximation theory, wavelets and applications (Maratea, 1994), 107-130, NATO Adv. Sci. Inst. Ser. C: Math. Phys. Sci., 454, Kluwer Acad. Publ., Dordrecht, 1995. MR 1340886

[15] M. A. Dritschel and J. Rovnyak, Operators on indefinite inner product spaces, in Lectures on operator theory and its applications (Waterloo, ON, 1994), 141-232, Fields Inst. Monogr., 3, Amer. Math. Soc., Providence, RI, 1996. MR 1364446

[16] A. Gheondea, On the geometry of pseudoregular subspaces of a Kreĭn space, in Spectral theory of linear operators and related topics (Timişoara/Herculane, 1983), 141-156, Oper. Theory Adv. Appl., 14, Birkhäuser, Basel, 1984. MR 0789614

[17] J. I. Giribet, A. Maestripieri and F. Martínez Pería, Abstract splines in Krein spaces, J. Math. Anal. Appl. 369 (2010), no. 1, 423-436. MR 2643880

[18] J. I. Giribet, A. Maestripieri and F. Martínez Pería, A geometrical approach to indefinite least squares problems, Acta Appl. Math. 111 (2010), no. 1, 65-81. MR 2653050

[19] J. I. Giribet, A. Maestripieri and F. Martínez Pería, Indefinite least-squares problems and pseudo-regularity, J. Math. Anal. Appl. 430 (2015), no. 2, 895-908. MR 3351987 
[20] B. Hassibi, A. H. Sayed and T. Kailath, Linear estimation in Kreĭn spaces. I. Theory, IEEE Trans. Automat. Control 41 (1996), no. 1, 18-33. MR 1372613

[21] B. Hassibi, A. H. Sayed and T. Kailath, Linear estimation in Krĕn spaces. II. Applications, IEEE Trans. Automat. Control 41 (1996), no. 1, 34-49. MR 1372614

[22] B. Hassibi, A. H. Sayed and T. Kailath, Indefinite-quadratic estimation and control. A unified approach to $H^{2}$ and $H^{\infty}$ theories, SIAM Studies in Applied Mathematics, 16, Society for Industrial and Applied Mathematics (SIAM), Philadelphia, PA, 1999. MR 1677962

[23] S. Izumino, The product of operators with closed range and an extension of the reverse order law, Tohoku Math. J. (2) 34 (1982), no. 1, 43-52. MR 0651705

[24] P.-J. Laurent, Approximation et optimisation, Collection Enseignement des Sciences, No. 13, Hermann, Paris, 1972. MR 0467080

[25] G. Loosli, S. Canu and C. S. Ong, Learning SVM in Krĕ̌n spaces, IEEE Trans. Pattern Anal. Mach. Intell. 38 (2016), 1204-1216.

[26] M. Z. Nashed, Inner, outer, and generalized inverses in Banach and Hilbert spaces, Numer. Funct. Anal. Optim. 9 (1987), no. 3-4, 261-325. MR 0887072

[27] H. Patel, Solving the indefinite least squares problems, Thesis (Ph.D.)-The University of Manchester (United Kingdom). ProQuest LLC, Ann Arbor, MI, 2002. MR 3819009

[28] J. Rovnyak, Methods of KreĬn space operator theory, in Interpolation theory, systems theory and related topics (Tel Aviv/Rehovot, 1999), 31-66, Oper. Theory Adv. Appl., 134, Birkhäuser, Basel, 2002. MR 2002586

[29] A. Sard, Optimal approximation, J. Functional Analysis 1 (1967), 222-244. MR 0233134

[30] A. H. Sayed, B. Hassibi and T. Kailath, Inertia conditions for the minimization of quadratic forms in indefinite metric spaces, in Recent developments in operator theory and its applications (Winnipeg, MB, 1994), 309-347, Oper. Theory Adv. Appl., 87, Birkhäuser, Basel, 1996. MR 1399368

\section{S. Gonzalez Zerbo}

Departamento de Matemática, Facultad de Ingeniería - Universidad de Buenos Aires, and Instituto Argentino de Matemática "Alberto P. Calderón” (CONICET), Saavedra 15, (1083) Buenos Aires, Argentina

sgzerbo@fi.uba.ar

\section{A. Maestripieri}

Departamento de Matemática, Facultad de Ingeniería - Universidad de Buenos Aires, and Instituto Argentino de Matemática “Alberto P. Calderón” (CONICET), Saavedra 15, (1083) Buenos Aires, Argentina

amaestri@fi.uba.ar

F. Martínez Pería

Centro de Matemática de La Plata (CMaLP) - FCE-UNLP, La Plata, Argentina, and Instituto Argentino de Matemática "Alberto P. Calderón" (CONICET), Saavedra 15, (1083) Buenos Aires, Argentina

francisco@mate.unlp.edu.ar

Received: March 25, 2020

Accepted: September 15, 2020 